\title{
Conflicting Readmission Rate Trends in a High-Risk Population: Implications for Performance Measurement
}

\author{
C. Annette DuBard, MD, MPH, Julie C. Jacobson Vann, $\mathrm{PhD}^{2}$, and Carlos T. Jackson, $\mathrm{PhD}^{1}$
}

\begin{abstract}
The 30-day readmission rate is a common performance indicator for hospitals and accountable care entities. There is reason to question whether measuring readmissions as a function of hospital discharges is an appropriate measure of performance for initiatives that aim to improve overall cost and quality outcomes in a population. The objectives of this study were to compare trends in 30-day readmission rates per discharge to population-based measures of hospital admission and readmission frequency in a high-risk statewide Medicaid population over a 5-year period of quality improvement and care management intervention. Further, this study aimed to examine case-mix changes among hospitalized beneficiaries over time. This was a retrospective analysis of North Carolina Medicaid paid claims 2008 through 2012 for beneficiaries with multiple chronic or catastrophic conditions. Thirty-day readmission rates per discharge trended upward from $18.3 \%$ in 2008 to $18.7 \%$ in 2012 . However, the rate of 30-day readmissions per 1000 beneficiaries declined from 123.3 to 110.7 . Overall inpatient admissions per 1000 beneficiaries decreased from 579.4 to 518.5. The clinical complexity of hospitalized patients increased over the 5-year period. Although rates of hospital admissions and readmissions fell substantially in this high-risk population over 5 years, the 30-day readmission rate trend appeared unfavorable when measured as a percent of hospital discharges. This may be explained by more complex patients requiring hospitalization over time. The choice of metrics significantly affects the perceived effectiveness of improvement initiatives. Emphasis on readmission rates per discharge may be misguided for entities with a population health management focus. (Population Health Management 2015;18:351-357)
\end{abstract}

\section{Introduction}

$\mathbf{H}$ OSPITAL READMISSION IS OFTEN an undesirable outcome from the perspectives of patient-centered care, cost, and quality. National data show that approximately $14 \%$ of adults discharged from a hospital are readmitted within 30 days. ${ }^{1}$ Readmissions are even more common among Medicare beneficiaries and Medicaid beneficiaries with disabilities, where historically $20 \%$ and $16 \%$ of discharged patients, respectively, return within 30 days. ${ }^{2,3}$ A growing body of evidence is demonstrating that transitional care coordination interventions can effectively reduce hospital readmission in the first 30 days after discharge, ${ }^{4,5,6}$ as well as longer term readmission and overall hospitalization rates in targeted populations. $6,7,8,9$

As reducing hospital readmissions has been identified as a key health care improvement strategy, readmission measures are increasingly being integrated into policy frame- works and contractual performance standards. The Centers for Medicare \& Medicaid Services (CMS) has been at the forefront of this effort, beginning in 2009 with the public reporting of readmission rates for most acute care hospitals in the United States, followed by payment reductions to hospitals with higher-than-expected readmission rates starting in October 2012. In these initiatives, the hospital discharge is the unit of analysis and the discharging hospital is held primarily accountable for risk-standardized readmission rates per discharge. ${ }^{10}$ The all-cause readmission rate, measuring readmissions per discharge, has been further applied by CMS in the Community-based Care Transitions Program, in which participating community-based organizations aim to collaboratively reduce readmissions, ${ }^{11}$ and as one of 33 performance measures for Accountable Care Organizations (ACOs) in the Medicare Shared Savings Program. Beyond these Medicare-specific programs, readmission rates calculated as a percent of hospital discharges

\footnotetext{
${ }^{1}$ Community Care of North Carolina, Raleigh, North Carolina.
}

${ }^{2}$ American Institutes for Research, Chapel Hill, North Carolina. 
have now been adopted as a Medicaid adult core quality measure, ${ }^{12}$ as a health plan quality measure under the National Committee for Quality Assurance Healthcare Effectiveness Data and Information Set, ${ }^{13,14}$ and into private sector initiatives including performance contracts with provider entities or ACOs. ${ }^{15,16}$

Conceptually, the goal of reducing the rate at which hospital-discharged patients are readmitted may be in conflict with the broader goal of reducing avoidable hospital utilization in the population overall under an accountable care framework. Chronically ill beneficiaries, particularly those with multiple chronic conditions, account for $98 \%$ of Medicare readmissions. ${ }^{17}$ Because these beneficiaries have a chronic, persistent risk of decompensation or acute exacerbation of chronic disease requiring hospitalization, interventions that improve access to primary care, quality of chronic disease care, and coordination among care providers should be expected to reduce the frequency of inpatient admissions in a well-managed population over time. Conceivably, that would lead to a shift in the inpatient demographics, with only the "sickest of the sick," those at highest risk of readmission, requiring hospitalization. Is it possible, then, that improving the overall care of patients with chronic conditions will paradoxically increase the hospital readmission rate by altering the risk profile of those who require hospital admission in the first place?

The experience of the North Carolina (NC) Medicaid program provides a unique opportunity to examine this question. Beginning in late 2007, NC expanded statewide enrollment of elderly and disabled Medicaid beneficiaries into the Community Care of North Carolina (CCNC) enhanced medical home model of care. ${ }^{18,19}$ Under CCNC management, which links recipients to a primary care medical home and provides community-based infrastructure for quality improvement and multidisciplinary care management for high-risk patients, NC saw a substantial decrease in inpatient utilization and total cost of care per beneficiary from 2007 to $2011 .^{20}$ CCNC's transitional care program, which expanded gradually since its inception in 2008 and currently provides care management support after hospital discharge to more than 2600 Medicaid recipients per month, is considered to be a key contributor to this success. ${ }^{21}$ In a prior study, this transitional care program was shown to substantially increase time to first, second, and third readmission among patients with multiple chronic conditions, with much of the benefit realized beyond the first 30 days. $^{8}$

The present study aimed to examine 30-day all-cause readmission rate trends among NC Medicaid beneficiaries

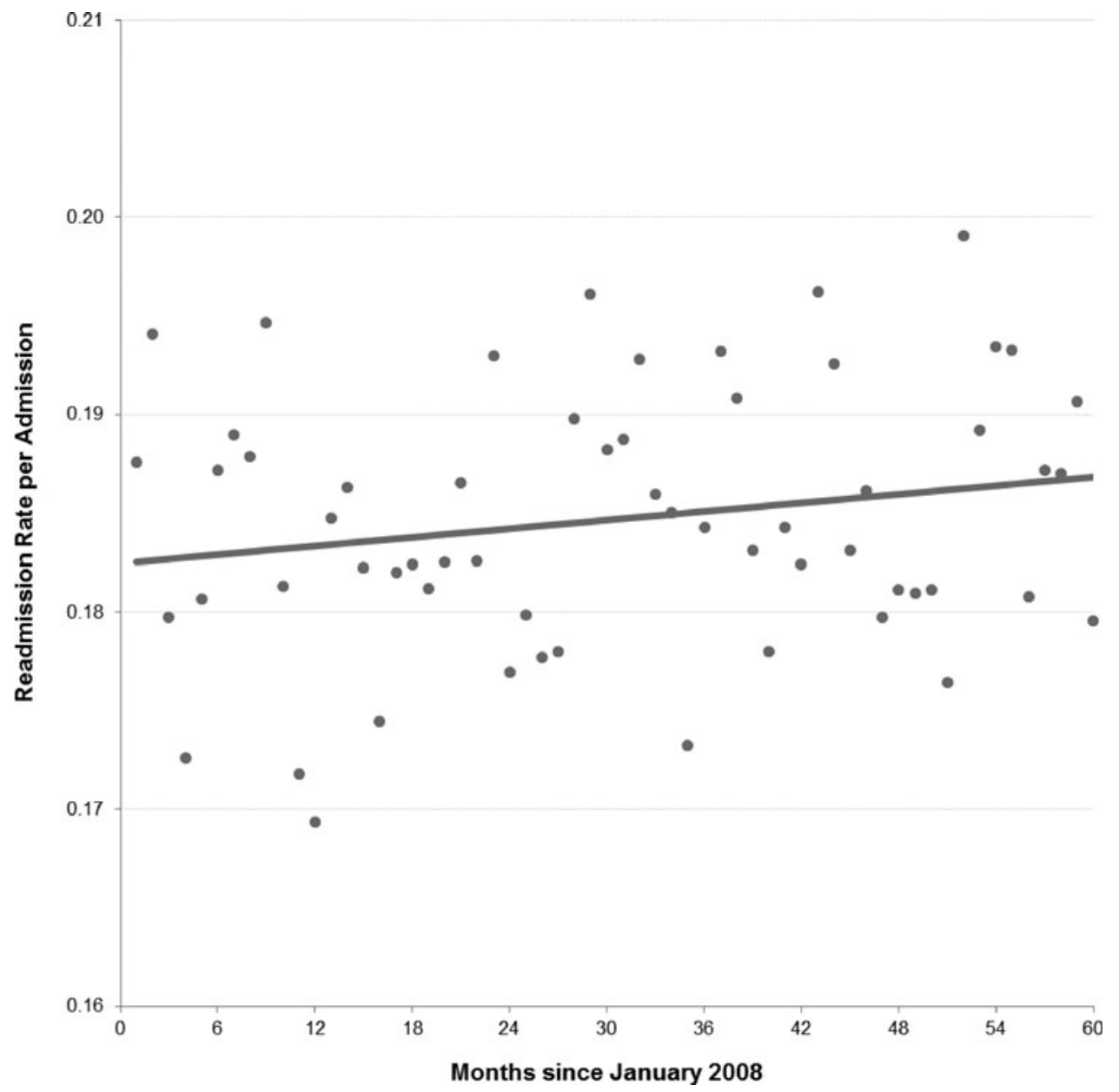

FIG. 1. 30-Day readmission rate per admission over time; North Carolina Medicaid beneficiaries with multiple chronic conditions. 
with multiple chronic conditions between 2008 and 2012 . The study reports readmission rates measured as a function of hospital discharges, following the logic most commonly deployed in performance measurement nationally, as well as the population-based measures of 30-day readmission rate per beneficiary-month and overall inpatient rate per beneficiary-month. This study further aimed to describe case-mix trends and inherent readmission risk among hospitalized beneficiaries to test the hypothesis that the risk profile of hospitalized patients will increase over time in a managed population.

\section{Methods}

The data for this study came from paid Medicaid claims data during January 1, 2008, and December 31, 2012. Analyses were limited to all NC Medicaid recipients identified as having multiple chronic or catastrophic conditions (MCCs) as defined by 3M Health Information Systems Clinical Risk Group methodology. ${ }^{22}$ Recipients dually eligible for Medicaid and Medicare were excluded because of lack of complete Medicare claims data. All inpatient visits were included for this population. Inpatient admissions that occurred within 30 days of a previous discharge were counted as a readmission, regardless of the reason for readmission; same-day hospital transfers were not counted as readmissions. The denominator for per beneficiary measures included all months in which recipients with MCCs were enrolled in NC Medicaid during the year.
To examine changes in case mix of hospitalized beneficiaries over time, hospital discharges were categorized according to the beneficiary's 3M Aggregated Clinical Risk Group (ACRG). To calculate benchmark readmission rates for each of these 18 clinical risk groups, the study team examined all hospitalizations from January 1, 2008, to December 31, 2012, dividing the number of readmissions within 30 days by the number of index discharges to compute an average readmission rate for the 5-year period.

Sensitivity analyses examined trends in 90-day readmission rates per discharge and 90-day readmissions per beneficiary-months. Findings were similar to the 30-day readmission results and not presented here for the sake of brevity.

\section{Results}

The number of individuals with MCCs covered under NC Medicaid increased from 127,951 in 2008 to 149,942 in December 2012. Total MCC beneficiary-months were 1,535,407 in $2008 ; 1,679,263$ in $2009 ; 1,763,091$ in $2010 ; 1,809,051$ in 2011; and 1,799,298 in 2012. Total hospital discharges for MCC beneficiaries numbered 74,132 in 2008; 77,681 in 2009; 79,354 in 2010; 82,699 in 2011; and 77,744 in 2012. The rate of readmission within 30 days of discharge fluctuated widely on a month-to-month basis, with an overall upward trend between $18 \%$ and $19 \%$ over the 5-year period (Fig. 1).

On a per-beneficiary basis, the rate of 30-day readmissions trended in the opposite direction, declining from

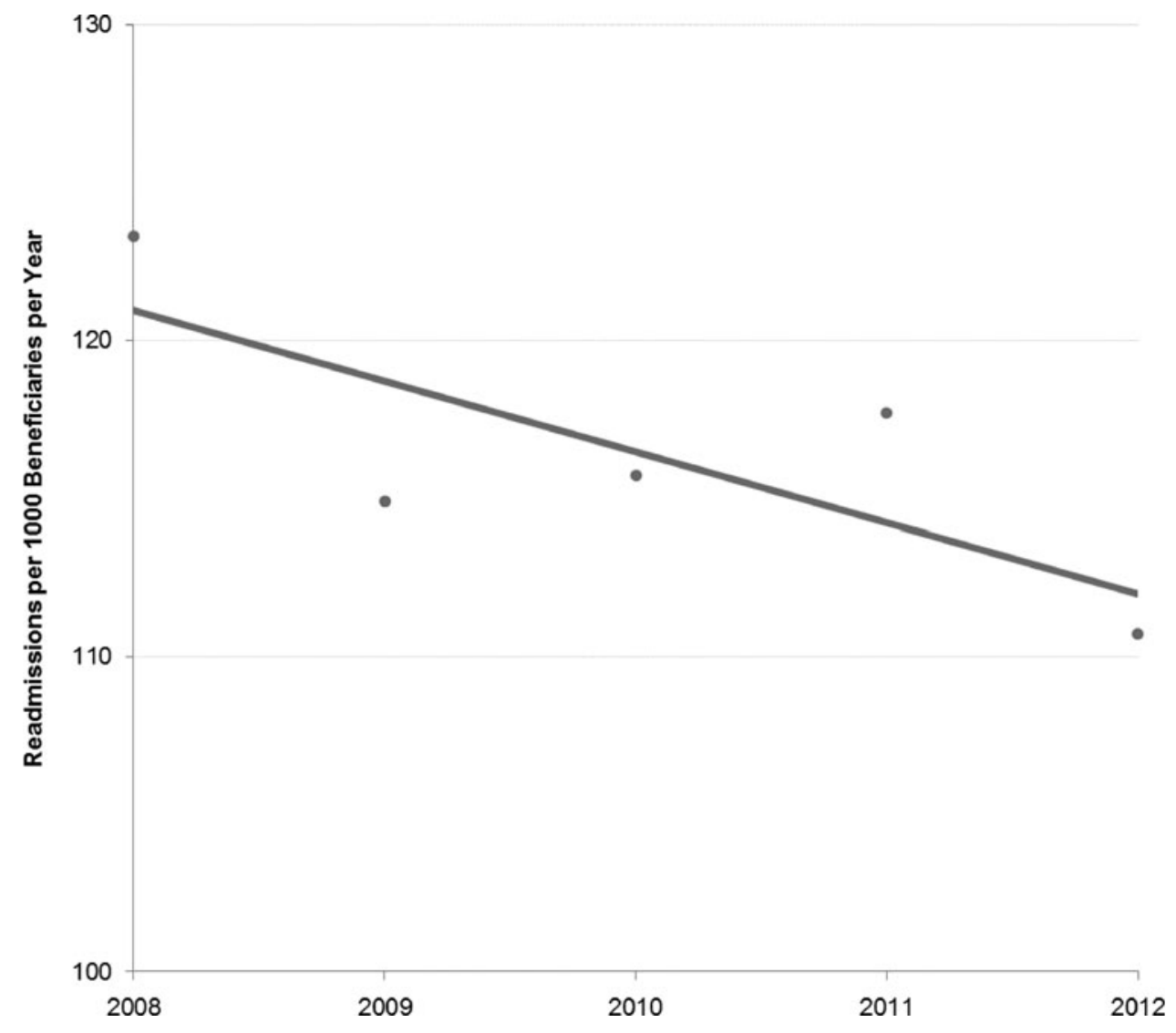

FIG. 2. 30-Day readmission rates per beneficiary over time; North Carolina Medicaid beneficiaries with multiple chronic conditions 
123.3 to 110.7 readmissions per 1000 beneficiaries per year from 2008 to 2012 (Fig. 2). Overall inpatient admissions per 1000 beneficiaries similarly decreased, from 579.4 in 2008 to 518.5 in 2012 (Fig. 3).

Benchmark readmission rates, based on the overall rate of readmission within 30 days of discharge for beneficiaries in each of 18 clinical risk groups over the 5-year period, ranged from 5.3\% to 41.8\% (Table 1). Between 2008 and 2012, the distribution of admissions shifted toward clinical risk groups with higher readmission risk. The proportion of admissions for patients in 10 risk groups with benchmark readmission rates $<20 \%$ remained stable or declined, while the proportion of admissions for patients in 7 of 8 risk groups with benchmark readmission rates $>20 \%$ increased. This resulted in an overall increase in expected readmission rate from $18.6 \%$ to $19.2 \%$. Logistic regression analysis demonstrated a statistically significant increase in the risk of readmission for hospitalized patients over time (Wald statistic $=11.56, P=.001)$ with the risk increasing an average of $.15 \%$ each year.

Note that the study team additionally examined benchmark readmission rates as a function of the Diagnosis Related Group (DRG) of the index hospitalization, following the same methodology described for ACRGs, and found a similar trend of higher-risk DRGs making up a larger proportion of the total admissions during each subsequent year.

\section{Discussion and Conclusions}

This study compared 2 different approaches for measuring 30-day readmission rates - per discharge and per beneficiaryin the context of a large-scale population health management initiative for Medicaid beneficiaries with chronic conditions. From 2008 to 2012, the proportion of beneficiaries who experienced a 30 -day readmission declined by $10.2 \%$, from 123.3 to 110.7 readmissions per 1000 beneficiaries per year, concomitant with a $10.5 \%$ decline in overall inpatient admission rates from 579.4 to 518.5 per 1000 beneficiaries per year. When presented as a percent of hospital discharges, however, as commonly deployed in health system or health plan performance measurement, readmission rates trended in the opposite direction-increasing from an average of $18.3 \%$ in 2008 to $18.7 \%$ in 2012 .

Analysis of the clinical complexity of hospitalized beneficiaries, which demonstrated a shift toward greater complexity and greater associated risk of readmission over the 5-year period, provides a possible explanation for these opposing trends. If improvements in access to care, chronic disease management, and care coordination successfully decrease hospitalization rates among chronically ill beneficiaries over time, it is reasonable to expect that those individuals who do require hospitalization will have greater clinical complexity or more advanced illness. The likelihood that

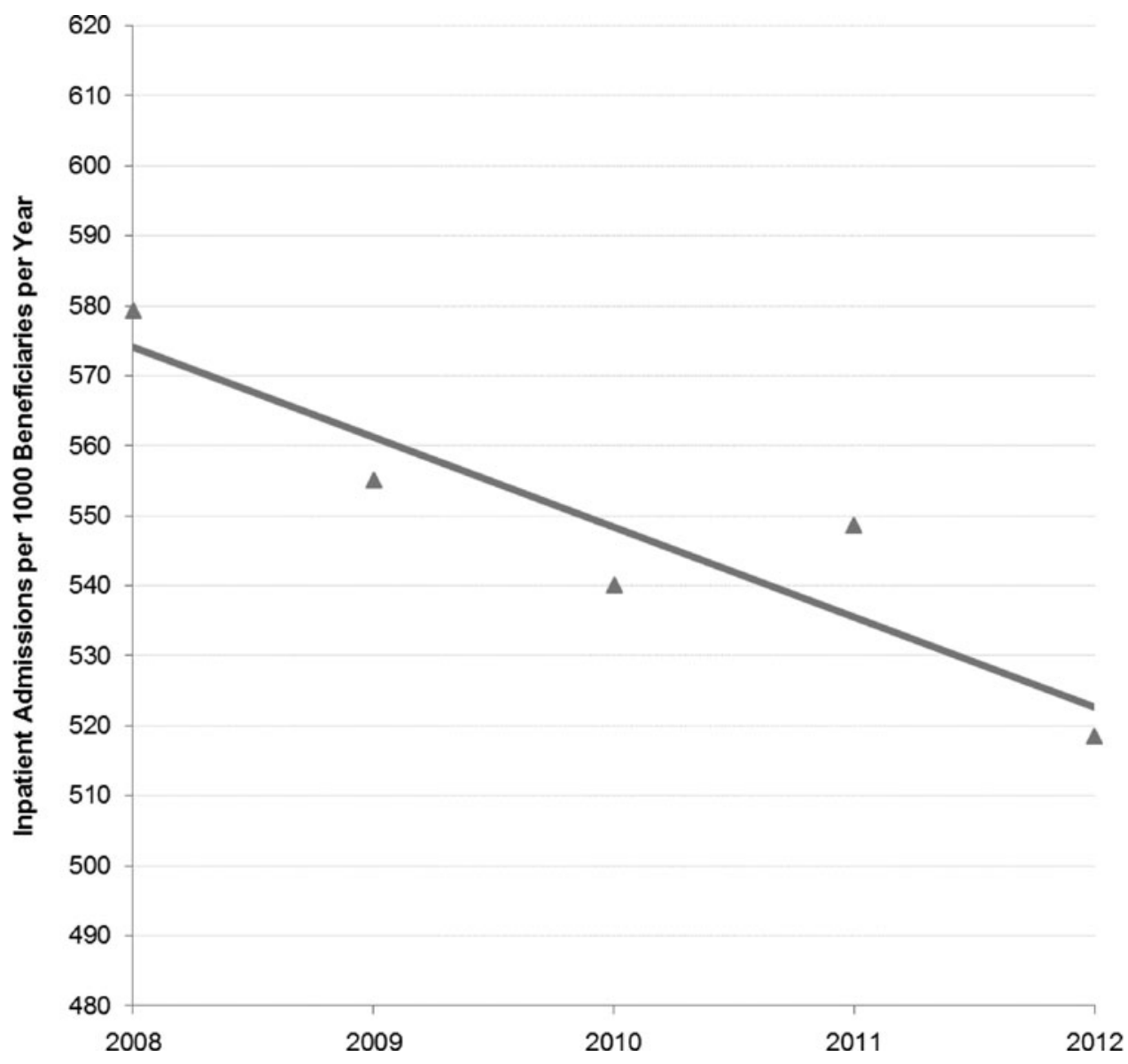

FIG. 3. Inpatient admission rates per beneficiary over time; North Carolina Medicaid beneficiaries with multiple chronic conditions. 


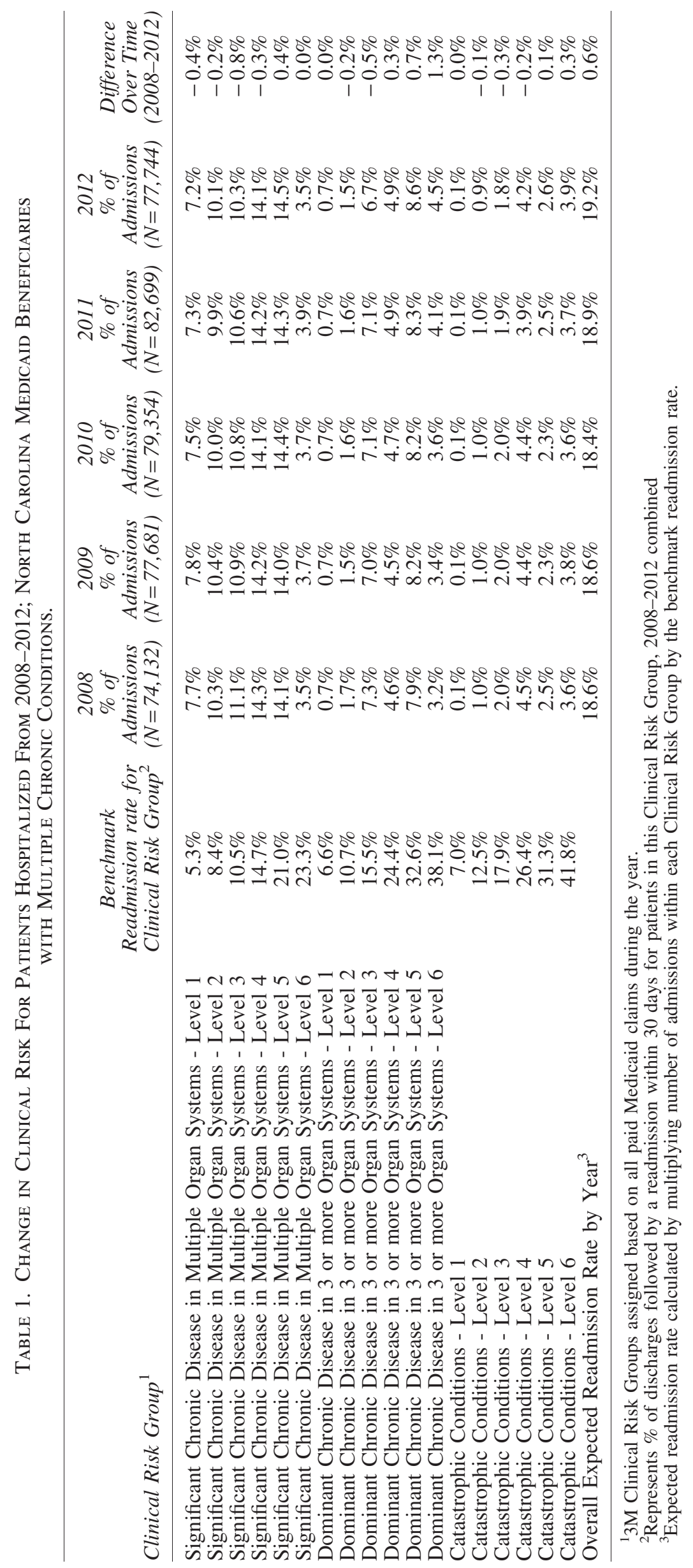


hospitalized patients will require readmission within 30 days can be expected to rise accordingly.

Opposing trends in overall admission rates and 30-day readmissions per discharge have been observed in other population-based care improvement initiatives. In a recent evaluation of 14 Quality Improvement Organization-facilitated community-wide initiatives to improve care transitions for Medicare beneficiaries, declines in overall readmission and admission rates were reported in the target population, despite no change in the rate of all-cause 30-day readmissions as a percentage of hospital discharges. ${ }^{4}$ A similar pattern was reported in the evaluation of the Medicare Physician Group Practice demonstration, Medicare's first pay-for-performance initiative to incentivize quality improvement and cost efficiency at the level of the physician group practice. Although the number of hospitalizations per beneficiary and share of beneficiaries with 1,2, and 3 discharges fell over the 5-year performance period, there was a slight rise in the rate of 30day readmissions per discharge. ${ }^{23}$

The present study augments prior studies that have identified weak or inverse associations between the 30-day readmission rate and other measures of health system performance. The 30-day readmission rate has been shown to be weakly or inversely associated with the initial hospital length of stay, inpatient days over an episode of care, Medicare's Hospital Compare process quality measures, and mortality. ${ }^{10,24,25,26}$ Alternative methodologies for measuring readmissions per discharge — such as the All-Cause Readmission rate index from United Health Group, CMS 30-day readmission rates from Hospital Compare, and the $3 \mathrm{M}$ Potentially Preventable Readmission Rate-also have been shown to correlate poorly with each other, leading to very different hospital performance rankings. ${ }^{27,28}$ As health systems increasingly participate in financial arrangements that hold them more accountable for the totality of patient experience, costs, and outcomes for a population, this study's findings should raise additional concerns about the potential for a chosen measure to provide an incomplete or misleading picture of health system performance. When tracking readmissions per discharge, robust methods for risk adjustment must be incorporated to allow not only for crosssectional comparisons to other entities, but for longitudinal observations within a target population.

NC's large-scale initiative to improve the care of chronically ill Medicaid beneficiaries provided a sizable high-risk population in which to observe admission and readmission trends over 5 years of intervention, but generalizability to non-Medicaid or lower risk populations may be limited. This study's observation that the clinical complexity of hospitalized beneficiaries increased over time was based on claims data, and it is possible that changes in provider coding behaviors to optimize billing may have contributed. However, a shift in case mix was not found within the study population as a whole, which suggests that any coding bias may be negligible.

The choice of health systems metrics and the resulting ratings or rankings of these entities can affect reimbursement levels, the reputation of an organization or system, and decisions about whether or not to continue or modify quality improvement and care coordination initiatives. For high-risk individuals with chronic care needs, who account for the vast majority of hospital readmissions, singular focus on short-term readmission rates would be misguided. To optimize care and outcomes in this population, it will be important to take the long view.

\section{Author Disclosure Statement}

Drs. DuBard, Vann, and Jackson declared no conflicts of interest with respect to the research, authorship, and/or publication of this article.

The authors received the following financial support: This work was supported in part by a grant from the North Carolina Healthcare Quality Alliance and the North Carolina Department of Health and Human Services, Office of Rural Health and Community Care.

\section{References}

1. Hines AL, Barrett ML, Jiang HJ, Steiner CA. Conditions with the largest number of adult hospital readmissions by payer, 2011. Healthcare Cost and Utilization Project. Statistical Brief \#172. April 2014. http://www.hcup-us.ahrq .gov/reports/statbriefs/sb172-Conditions-Readmissions-Payer .pdf. Accessed August 29, 2014.

2. Jencks SF, Williams MV, Coleman EA. Rehospitalizations among patients in the Medicare fee-for-service program. N Engl J Med. 2009;360:1418-1428.

3. Gilmer T, Hamblin A. (2010). Hospital readmissions among Medicaid beneficiaries with disabilities: identifying targets of opportunity. Faces of Medicaid Data Brief. Center for Health Care Strategies, Inc. December 2010. http://www.chcs.org/media/CHCS_readmission_101215b.pdf. Accessed August 29, 2014.

4. Brock J, Mitchell J, Irby K, et al. Association between quality improvement for care transitions in communities and rehospitalizations among Medicare beneficiaries. JAMA. 2013; 309:381-391.

5. Jack BW, Chetty VK, Anthony D, et al. A reengineered hospital discharge program to decrease rehospitalization: a randomized trial. Ann Intern Med. 2009;150:178-188.

6. Coleman EA, Parr C, Chalmers S, Min S. The care transitions intervention: results of a randomized controlled trial. Arch Intern Med. 2006;166:1823-1828.

7. Verhaegh KJ, MacNeil-Vroomen JL, Eslami S, Geerlings SE, De Rooij SE, Buurman BM. Transitional care interventions prevent hospital readmissions for adults with chronic illnesses. Health Aff (Millwood). 2014;33:1531-1539.

8. Jackson CT, Trygstad TK, DeWalt DA, DuBard CA. Transitional care cut hospital readmissions for North Carolina Medicaid patients with complex chronic conditions. Health Aff (Millwood). 2013;32:1407-1415.

9. Naylor MD, Aiken LH, Kurtzman ET, Olds DM, Hirschman KB. The importance of transitional care in achieving health reform. Health Aff (Millwood). 2011;30:746-754.

10. Press MJ, Scanlon DP, Ryan AM, et al. Limits of readmission rates in measuring hospital quality suggest the need for added metrics. Health Aff (Millwood). 2013;32:10831091.

11. Centers for Medicare \& Medicaid Services. Communitybased care transitions program. http://innovation.cms.gov/ initiatives/CCTP/index.html. Accessed September 7, 2014.

12. Center for Medicaid and CHIP Services; Centers for Medicare and Medicaid Services. Core Set of Health Care Quality Measures for Adults Enrolled in Medicaid (Medicaid Adult Core Set): Technical Specifications and Resource Manual for Federal Fiscal Year 2014 Reporting. May 
2014. http://www.medicaid.gov/Medicaid-CHIP-ProgramInformation/By-Topics/Quality-of-Care/Downloads/MedicaidAdult-Core-Set-Manual.pdf. Accessed August 29, 2014.

13. National Committee for Quality Assurance. 2012 Insights for Improvement. Reducing Readmissions: Measuring Health Plan Performance. 2012. http://www.ncqa.org/ portals/0/Publications/2012\%20BI_NCQA\%20ReAdMi\%20_ Pub.pdf. Accessed August 29, 2014.

14. Morgan A, Khan A, Amin T. Challenges in evaluating allcause hospital readmission measures for use as national consensus standards. Perm J. 2013;17(4):14-18.

15. Nowicki S, Zembroski D, Pickering L, Nobel J. Reducing Preventable Hospital Readmissions: A Multistakeholder Perspective. July 2012. http://www.nebgh.org/publications/ HospitalRedadmissionsReport_V2_SinglePages.pdf. Accessed on August 30, 2012.

16. United Healthcare. Quality of care guideline. Hospital readmissions. August 1, 2014. https://www.unitedhealthcareonline .com/ccmcontent/ProviderII/UHC/en-US/Assets/ProviderStatic Files/ProviderStaticFilesPdf/Tools\%20and\%20Resources/ Policies\%20and\%20Protocols/Medical\%20Policies/Medical\% 20Policies/Hospital_Readmissions.pdf. Accessed August 31, 2014.

17. Centers for Medicare \& Medicaid Services. Chronic Conditions among Medicare Beneficiaries. Chart book: 2012 Edition. http://www.cms.gov/Research-Statistics-Data-andSystems/Statistics-Trends-and-Reports/Chronic-Conditions/ Downloads/2012Chartbook.pdf. Accessed August 30, 2014.

18. Steiner BD, Denham AC, Ashkin E, Newton WP, Wroth T, Dobson LA. Community Care of North Carolina: improving care through community health networks. Ann Fam Med. 2008;6:361-367.

19. Dobson LA, Hewson DL. Community Care of North Carolina-an enhanced medical home model. N C Med J. 2009;70:219-224.

20. Fillmore H, DuBard CA, Ritter GA, Jackson CT. Health care savings with the patient-centered medical home: Community Care of North Carolina's experience. Popul Health Manag. 2013;17:141-148.

21. DuBard CA, Cockerham J, Jackson C. Collaborative accountability for care transitions: the Community Care of
North Carolina transitions program. N C Med J. 2012;73: 34-40.

22. Hughes JS, Averill RF, Eisenhandler J, et al. Clinical Risk Groups (CRGs): a classification system for risk-adjusted capitation-based payment and health care management. Med Care. 2004;42:81-90.

23. Kautter J, Pope GC, Leung M, et al. Evaluation of the Medicare Physician Group Practice Demonstration. Final Report. September 2012. http://www.cms.gov/Medicare/ Demonstration-Projects/DemoProjectsEvalRpts/Downloads/ PhysicianGroupPracticeFinalReport.pdf. Accessed August 29, 2014.

24. Kociol RD, Liang L, Hernandez AF, et al. Are we targeting the right metric for heart failure? Comparison of hospital 30-day readmission rates and total episode of care inpatient days. Am Heart J. 2013;165:987-994.

25. Stefan MS, Pekow PS, Nsa W, et al. Hospital performance measures and 30-day readmission rates. J Gen Intern Med. 2013;28:377-385.

26. Krumhotz HM, Lin Z, Keenan PS, et al. Relationship between hospital readmission and mortality rates for patients hospitalized with acute myocardial infarction, heart failure, or pneumonia. JAMA. 2013;309:587-593.

27. Boutwell AE, Jencks SF. STAAR Issue Brief: Reducing barriers to care across the continuum: measuring rehospitalizations at the state level. 2010. http://www.ihi.org/ Engage/Initiatives/Completed/STAAR/Documents/STAAR\% 20Issue \%20Brief\%20-\%20Data\%20and\%20Measurement .pdf. Accessed August 30, 2014.

28. Davies S, Saynina O, Schultz E, McDonald KM, Baker LC. Implications of metric choice for common applications of readmission metrics. Health Serv Res. 2013;48:1978-1995.

Address correspondence to: C. Annette DuBard, MD, MPH Community Care of North Carolina 2300 Rexwoods Drive, Suite 200 Raleigh, NC 27607

E-mail: adubard@n3cn.org 